\title{
Informação geral e atual e sua relação com a inteligência e a personalidade em crianças escolares ${ }^{1}$
}

\author{
General and current information and its relationship \\ with the student's intelligence and personality
}

\author{
Carmen FLORES-MENDOZA ${ }^{2}$ \\ Graciane Lopes JARDIM \\ Francisco José $\mathbf{A B A D}^{3}$ \\ Larissa Assunção RODRIGUES²
}

\begin{abstract}
Resumo
Investiga-se a relação entre inteligência, personalidade e nível de informação geral e atual de escolares do Estado de Minas Gerais. Duas amostras participaram do estudo: a primeira proveniente de escolas de três níveis de vulnerabilidade social de Belo Horizonte (MG) ( $n=600)$, e a segunda de escolas municipais da cidade de Perdões (MG) ( $n=215)$. Ambas as amostras foram submetidas ao teste Raven e ao Questionário de Informaçōes Gerais e Atuais. A segunda amostra realizou o Eysenck Questionnaire Personality e o subteste Informação do WISC-III. Os resultados mostraram uma associação consistente entre inteligência e o Questionário de Informações Gerais e Atuais, mesmo controlando-se o efeito da vulnerabilidade social das escolas $(r=0,431)$. Uma path analyses mostrou predição independente da inteligência $(0,430)$ e da dimensão Psicoticismo $(-0,18)$ sobre Questionário de Informações Gerais e Atuais, após controle da idade e da covariância entre os preditores. Conclui-se que a inteligência explica as diferenças de Questionário de Informações Gerais e Atuais duas vezes mais do que a personalidade.
\end{abstract}

Unitermos: Informação. Inteligência. Psicoticismo. Personalidade.

\begin{abstract}
The aim of this study was to investigate the relationship between intelligence, personality and the extent of general and current information of students in the state of Minas Gerais. Two sample groups participated. The first was composed of students from three socially vulnerable levels from the city of Belo Horizonte ( $n=600)$, and the second group came from public schools in the city of Perdões $(n=215)$. The Raven's Progressive Matrices Test and a General Information Questionnaire were applied for both samples. In addition, the Eysenck Personality Questionnaire and the WISC-III Information test were applied to the second sample. The results indicated a consistent relationship between intelligence and General Information Questionnaire, even after smoothing the effect of the social vulnerability of the schools $(r=0.431)$. A path analysis showed an independent effect of intelligence $(r=0.430)$ and of the Psychoticism dimension $(-0.18)$ on the General Information Questionnaire, even after the smoothing of age and co-variance between predictors. It may be concluded that intelligenceexplains General Information Questionnaire differences twice as much as does personality.
\end{abstract}

Uniterms: Information. Intelligence. Psychoticism. Personality.

URV

1 Apoio: Fundação de Amparo à Pesquisa do Estado de Minas Gerais (Fapemig-SHA-processo no 283/06).

2 Universidade Federal de Minas Gerais, Faculdade de Filosofia e Ciências Humanas, Departamento de Psicologia, Gab. 4042, Laboratório de Avaliação das Diferenças Individuais. Av. Antônio Carlos, 6627, 31270-901, Belo Horizonte, MG, Brasil. Corespondência para/Correspondence to: C. FLORES-MENDOZA. E-mail: <carmencita@fafich.ufmg.br>.

$\mathbf{3}$ Universidade Autónoma de Madri. Madri, Espanha. 
Em junho de 2007, um programa famoso da televisão brasileira selecionava entre jovens profissionais aquele que poderia ocupar o posto de sócio do empresário e então apresentador do programa. No entanto, um dos mais fortes candidatos foi demitido. Duas foram as razões: ter escrito errado o nome do empresário/apresentador e ter afirmado que a cidade de Santiago teria ultrapassado São Paulo como capital de negócios na América do Sul. O candidato a empresário desconhecia que em 2006 a balança comercial do Estado de São Paulo fora seis vezes superior ao do país vizinho. O caso acima é um exemplo de uma das exigências do mundo moderno, espeficamente do atual mercado de trabalho baseado em conhecimento: manter-se informado do que ocorre no país e fora dele, assim como saber usar as informações (O'Sullivan, 2002; Organization for Economic Co-Operation and Development, 2001).

Em nível coletivo são numerosos os exemplos do alto custo que a desinformação dos cidadãos acarreta para os países, principalmente quando ela se dá na área da saúde. Por tal razão, existem representantes da sociedade civil planetária - como o Communication Rights in the Information Society (CRIS) - que procuram, de forma louvável, assegurar o direito à informação dos indivíduos independentemente da sua origem étnica ou socioeconômica, defendem o uso democrático dos meios de comunicação e tentam garantir a liberdade de expressão. Diferentemente do que ocorria na sociedade industrial do século XIX, a partir da segunda metade do século XX, a informação atual e geral confere considerável vantagem e benefício aos indivíduos e sociedades que a detêm.

Mas o que é "informação"? Existem diversas formas de abordar seu significado. A informação pode ser entendida como um processo que provoca mudança no sistema de representações do receptor; como conhecimento, cuja relevância depende do nível prévio do receptor; e como objeto, cujas características físicas determinam sua qualidade informativa. Entre elas, segundo Oleto (2006), a definição mais utilizada é aquela que trata a informação como um dado ou fato sobre algo ou alguém, e que propicia o conhecimento.

Sabe-se que a informação se dissemina de forma diferenciada na população. Identificar os fatores que influenciam essa diferenciação tem sido objetivo da

162 psicologia científica. Nesse caso, a mais forte candidata para explicar a variação de informação entre as pessoas parece ter sido a inteligência, que apresenta um peso significativo nas pesquisas atuais (Lubinski, 2004). A inteligência é vista como um construto que mantém certa estabilidade ao longo da vida de um indivíduo e, principalmente, uma forte associação com a educação e outros fenômenos da vida, como, por exemplo, saúde, longevidade, ascensão profissional, ganho salarial (Brody, 1992; Deary, Whitman, Starr, Whalley \& Fox, 2004; Gotffredson, 2003; Jensen, 1998).

Segundo Jensen (1998), “Todo conhecimento que um indivíduo possui certamente foi adquirido através da aprendizagem. É obvio que existe um amplo intervalo de diferenças individuais na taxa de aprendizagem, tanto no que se refere à quantidade apreendida quanto no nível superior de complexidade e de abstração daquilo que pode ser apreendido". Portanto, não é sem razão que o conhecimento geral está sempre presente em testes tradicionais de inteligência, como as escalas Wechsler, com o subteste chamado Informação (Wechsler, 2002), ou a escala Stanford-Binet 5, com o teste Procedural Knowledge (Roid, 2003).

A literatura vem registrando, por outro lado, o poder preditivo de traços de personalidade nas diferenças de aprendizagem de nova informação em crianças e adolescentes. Os traços referem-se geralmente aos do modelo trifatorial de Eysenck como Psicoticismo-P, Extroversão-E, Neuroticismo-N ou aos do modelo dos Cinco Grandes Fatores como Neuroticismo-N, Extroversão-E, Abertura-O, Amabilidade-A e Conscienciosidade-C (Chamorro-Premuzic \& Furnham, 2003; De Raad \& Schouwenburg, 1996; Guerin, Gottfried, Oliver \&Thomas, 1994; Martin, Drew, Gaddis \& Moseley, 1988).

Há discrepâncias, entretanto, sobre qual a dimensão de personalidade que melhor prediz as diferenças na aprendizagem de informação, seja ela formal (contexto escolar) ou informal (quotidiano). Heaven, Mak, Barry e Ciarrochi (2002), por exemplo, encontraram que $\mathrm{P}-, \mathrm{A}+\mathrm{e} \mathrm{C}+$ se correlaciona com o desempenho escolar, mas em outro estudo, Heaven, Ciarrochi e Vialle (2007) encontraram apenas uma modesta contribuição de $\mathrm{P}$. Já Slobodskaya, Safronova e Windle (2005) não encontraram qualquer associação significativa entre personalidade e rendimento acadêmico.

Quando se trata de comparar o poder preditivo da inteligência com o da personalidade, infelizmente, 
encontra-se uma menor frequência de investigações. Entre os registrados, Chamorro-Premuzic, Furnham e Ackerman (2006), ao estudar o conhecimento geral de universitários britânicos, mostraram que as medidas de inteligência responderam por 26\% da variância, enquanto $\mathrm{N}$ e E responderam por apenas 5\%.

Laidra, Pullman e Allik (2007) estudaram o desempenho acadêmico (média das notas de todas as disciplinas) de 3618 estudantes da Estônia e encontraram que inteligência e personalidade foram responsáveis por 30\% da variância dos resultados, mas foi a inteligência a melhor preditora do rendimento escolar em todas as séries. Entre os fatores de personalidade, A foi o fator com melhor predição do desempenho escolar no ensino primário e o fator C na secundária.

Petrides, Chamorro-Premuzic, Frederikson e Furnham (2005) mediram habilidade verbal e desempenho escolar em 901 estudantes de ensino secundário. Encontraram uma forte associação entre essas variáveis $(0,84)$, enquanto o fator $P$ associou-se em -0,14 e o E, em $-0,25$.

A maioria dos estudos de inteligência, de personalidade ou de ambos considera como critério de desempenho acadêmico aquele relacionado a conteúdos escolares e que são medidos via nota, teste ou média das notas obtidas no ano. Trata-se, portanto, de um critério vinculado à aprendizagem ocorrida de maneira formal e que é imposta obrigatoriamente aos alunos. Pouco se sabe da influência da inteligência e da personalidade na aprendizagem de informação geral, isto é, naquele conhecimento que não necessariamente é parte rotineira das avaliações escolares ou de testes de conhecimento. Ou, ainda, na informação atual, isto é, na aquisição espontânea daquela informação que causa impacto na sociedade depois de disponibilizada pela mídia em geral e que permeia as conversações informais.

Entre os que estudam os fatores que envolvem o conhecimento geral e atual, têm-se os trabalhos de Beier e Ackerman (2001; 2003; 2005). Esses autores avaliaram o desempenho de adultos em conhecimentos gerais e específicos. No estudo de 2001, por exemplo, os autores encontraram que o composto de medidas de Gf (inteligência fluida) apresentou correlação entre 0,43 a 0,51 com conhecimento de Política/Economia e Ciência respectivamente; enquanto Gc (inteligência cristalizada) apresentou correlação entre 0,76 e 0,81. A idade associou-se também positivamente aos conhecimentos. A partir desses resultados, os autores realizaram path analysis para identificar o melhor preditor. Encontraram uma associação entre Gf e Gc de 0,68 e, portanto, nenhum dos dois modelos obteve adequado ajuste aos dados. Em outro estudo efetuado com sujeitos adultos em ambientes de aprendizagem livre e controlada, os autores encontraram o mesmo problema (Beier \& Ackerman, 2005). Nessa situação, é provável que a ausência de um modelo que represente $g$ (dada a alta relação entre Gf e Gc) poderia ter dificultado encontrar um maior ajuste aos dados. Tratando-se de personalidade, o estudo de 2001 mostrou que unicamente o fator O apresentou alguma predição.

Um estudo mais amplo foi feito por Hambrick, Pink, Meinz, Pettibone e Oswald (2008). Os autores investigaram o conhecimento anterior e conhecimento de eventos gerais e atuais com medidas cognitivas, de personalidade e de memória. Encontraram um efeito direto do fator $g(0,44)$ sobre o conhecimento anterior, enquanto o efeito de Gc e Gf (independente de $g$ ) foi de 0,52 e -0,17 respectivamente. O conhecimento anterior, por sua vez, teve um efeito direto sobre conhecimento atual $(0,73)$, isto é, quem sabia mais continuava sabendo mais. Em relação à personalidade, o fator "abertura intelectual" teve efeito direto no fator "interesse por eventos gerais" e este por sua vez em "aquisição de novas informações". Os dois últimos tiveram efeito em torno de 0,30 tanto no conhecimento anterior quanto no atual. Os autores interpretaram tais resultados como evidência de que Gc teria uma contribuição maior que Gf no conhecimento anterior e atual e que algumas dimensões não cognitivas relacionadas à personalidade também participariam. Contudo, apesar das interpretações dos autores sobre o maior efeito de Gc, surpreende observar o efeito negativo de Gf no conhecimento anterior $(-0,17)$. Tal resultado teoricamente é contraintuitivo, pois desconsidera a existência de uma associação extremamente alta entre g e Gf - aproximadamente de 0,95 (Jensen, 1998). Portanto, a extração de Gf independente de $g$ desperta dúvida sobre o que Gf representou no modelo dos autores.

Os resultados dos estudos sobre diferenças no conhecimento geral e atual são consistentes: a) existe uma influência da idade, b) a inteligência é uma das 
melhores variáveis preditoras e c) dimensões de personalidade também causam efeitos na aprendizagem, embora em menor intensidade.

Se desconhece se tais observações são também encontradas em população escolar. Nesse sentido, o presente estudo tenta verificar a relação entre o nível de informação atual e geral e as dimensões de inteligência e personalidade em duas amostras de escolares de Minas Gerais.

\section{Método}

\section{Participantes}

Participaram 815 escolares com idade entre $9 \mathrm{e}$ 15 anos, sendo 52,8\% do gênero feminino. Desse total, 600 eram provenientes de três escolas públicas de Belo Horizonte (MG) (idade média =9,47; Desvio-Padrão $\mathrm{DP}=0,50)$ e 215 de escolas públicas da cidade de Perdões (MG) (idade média de 11,56; DP=1,93). No que se refere às escolas de Belo Horizonte, elas foram selecionadas aleatoriamente a partir do cadastro de estabelecimentos de ensino (disponível no site www.inep.gov.br/ basica/curso/cadastroescolas). A seleção foi feita para cada nível de vulnerabilidade social (baixa, média e alta) do bairro em que as escolas estavam localizadas. A classificação da vulnerabilidade social advém de uma escala elaborada por Nahas, Ribeiro, Esteves, Moscovitch e Martins (2000) para a cidade de Belo Horizonte. As escolas da cidade de Perdões, por sua vez, constituíam as existentes no município. Os participantes da amostra de Belo Horizonte frequentavam a terceira e a quarta série do ensino fundamental, enquanto a de Perdões frequentava entre a terceira e a oitava série.

\section{Instrumentos e Procedimento}

Por razões de objetivos diferenciados nas avaliações das amostras e disponibilidade de tempo, material e de equipe de avaliação, as amostras foram submetidas a instrumentos diversos, havendo, em comum, entretanto, a aplicação do QIG. Assim, na amostra de escolares de Belo Horizonte, aplicou-se o teste das Matrizes Progressivas de Raven - Escala Colorida (Angelini, Alves, Custório, Duarte \& Duarte, 1999). Já na amostra de escolares da cidade de Perdões, aplicaram-se o teste das Matrizes - Escala Geral (Raven, 2001), o subteste Informação da Escala Verbal do WISC III (Weschler, 2002) e o Eysenck Personality Questionnaire (Eysenck\&Eysenck, 1975). Às duas amostras, administrou-se um Questionário de Informação Geral (QIG). As aplicações ocorreram entre abril e agosto de 2006.

O QIG foi construído com base em uma lista de perguntas sobre acontecimentos ocorridos no ano anterior à coleta de dados, isto é, 2005, e fartamente veiculados na mídia nacional, assim como em questões de cultura geral. Essa lista foi submetida a dois juízes, doutores em psicologia, com o intuito de verificar a representatividade das perguntas sobre informações gerais e não de resolução de problemas, esta última característica de um teste de inteligência, que, portanto, poderia redundar com o objetivo do teste Raven. Ao todo foram selecionadas 20 perguntas. Cada pergunta continha três alternativas de resposta, sendo uma a correta (Quadro 1).

Quadro 1. Exemplos de itens do Questionário de informação geral e atual.

\begin{tabular}{|c|c|c|}
\hline & Questões & Alternativas de resposta \\
\hline 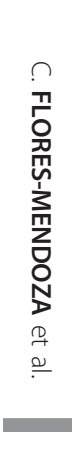 & $\begin{array}{l}\text { Qual a região americana devastada pelo furacão Katrina em } 28 \text { de agosto de } 2005 \text { ? } \\
\text { Qual o nome do novo papa nomeado em } 2005 . \\
\text { Qual famoso ditador árabe que está sendo julgado em Bagdá por crimes contra a humanidade? }\end{array}$ & $\begin{array}{l}\text { (X) Roberto Jefferson } \\
\text { ( ) José Dirceu } \\
\text { ( ) Antônio Palloci } \\
\text { ( ) Washington } \\
\text { ( ) New York } \\
\text { (X) New Orleans } \\
\text { (X) Bento XVI } \\
\text { ( ) João Paulo II } \\
\text { ( ) Pedro IV } \\
\text { ( ) Augusto Pinochet } \\
\text { ( ) Milosevic } \\
\text { (x) Saddam Hussein }\end{array}$ \\
\hline
\end{tabular}

*Questionário elaborado e administrado em 2006. 
Os instrumentos Raven, QIG e EQP-J foram aplicados de forma coletiva após assinatura do Termo de Consentimento Informado pela escola e pelos pais conforme orientação do Conselho Nacional de Saúde (item IV da resolução196/96). O subteste Informação da escala verbal do WISC III foi aplicado individualmente conforme orientação do manual do teste.

\section{Análises preliminares}

A confiabilidade da utilização das escalas foi obtida mediante o índice de consistência interna (Alpha de Cronbach). O valor obtido foi de 0,85 para a Escala Colorida de Raven (BH); 0,93 para a Escala Geral (Perdões); 0,80 para o subteste Informação (Perdões) e de 0,73 para o QIG (BH e Perdões). Tais resultados indicam, portanto, que as respostas aos instrumentos foram homogêneas.

Com relação à confiabilidade e à validade fatorial do EPQ-J, o Laboratório de Avaliação das Diferenças Individuais da Universidade Federal de Minas Gerais (UFMG) vem realizando estudos sistemáticos sobre o instrumento (Mansur-Alves, 2007). Com amostras maiores, as investigações apontam índices alpha de 0,65, 0,78, 0,72 e 0,79 para os fatores de Extroversão, Neuroticismo, Psicoticismo e Sinceridade respectivamente. A técnica conhecida como Comprehensive Exploratory Factor Analysis com rotação target ou chamada também de rotação procrusteana (Browne, Cudeck, Tateneni \& Mels, 2004) mostrou parâmetros de ajuste adequados para o modelo de quatro fatores: $\chi^{2}=3195,49 ; d f=2,012 ; \chi^{2}=1,588 ; R M S E A=0,038$.

No tratamento simultâneo das variáveis de inteligência, personalidade e informação geral se utilizou a path analysis, uma extensão do modelo de regressão que verifica o ajuste da matriz de correlação versus dois ou mais modelos causais que estão sendo comparados pelo pesquisador (Bryman \& Cramer, 1990).

\section{Resultados}

\section{Amostra Belo Horizonte}

Encontrou-se uma associação moderada entre idade e o teste Raven ( $r=0,103 ; p=0,000)$ assim como entre aquela e o QIG ( $r=0,244 ; p=0,000)$, apesar da restrição de idade (nove e 10 anos). Assim sendo, decidiu-se pela tipificação dos escores a fim de eliminar efeitos desenvolvimentais. A partir da aplicação coletiva, os dados brutos do teste Raven e do QIG foram então convertidos a escore-Z controlando-se a idade cronológica. Os escores de Raven foram transformados em QI mediante a fórmula: [z*15] +100 (Tabela 1).

A correlação entre o QI e os escores tipificados em $z$ do QIG foi de 0,447 ( $p<0,001$ ): uma associação moderada entre inteligência e nível de informação geral. A correlação foi positiva e estatisticamente significativa $(p<0,001)$ para ambos os genêros $(r=0,469$ para meninas e $r=0,423$ para meninos). Uma comparação de médias pelo teste ANOVA, no entanto, mostrou diferenças significativas entre escolas com respeito ao teste Raven [(2, $597)=10,61 ; p<0,001]$ e ao QIG $[(2,571)=11,78 ; p<0,001]$. Isso significa haver um efeito nos resultados do QIG do nível de vulnerabilidade social da escola. A correlação entre vulnerabilidade da escola (variável ordinal em que a pontuação maior é dada à zona com menor vulnerabilidade social) e o QIG, pelo método Kendall's tau_b, foi de 0,124 ( $p<0,001)$. Provavelmente, quanto menor o risco social da escola, maior o nível de informação geral e atual das crianças.

Com base nesses resultados, realizou-se uma correlação parcial entre as variáveis inteligência e QIG com controle do nível de vulnerabilidade social da escola. $O$ valor se manteve quase inalterado $(r=0,431$; $p<0,001)$. Por outro lado, a correlação parcial entre vulnerabilidade social da escola e o QIG, controlando-se a inteligência, foi de 0,142 ( $p<0,001)$. A inteligência manteve, portanto, uma associação com o critério externo superior à da vulnerabilidade social.

\section{Amostra Perdões}

A Tabela 2 mostra as estatísticas descritivas do desempenho da amostra de Perdões em todas as medidas utilizadas.

Tabela 1. Distribuição da pontuação bruta no QIG em cada faixa de QI para amostra de Belo Horizonte $(n=600)$.

\begin{tabular}{lrrc}
\hline \multirow{2}{*}{ Faixas de Q| } & \multirow{2}{*}{ Q|G } \\
\cline { 3 - 4 } & & Média & Desvio-Padrão \\
\hline $55-70$ & 2,82 & 4,46 & 1,99 \\
$71-85$ & 11,29 & 5,98 & 2,41 \\
$86-100$ & 33,00 & 7,37 & 2,91 \\
$101-115$ & 35,00 & 8,47 & 2,77 \\
$116-130$ & 15,25 & 9,56 & 2,76 \\
$131-145$ & 2,63 & 11,07 & 2,46 \\
\hline
\end{tabular}

"Resultados do teste Raven (Escala Colorida) transformados em QI. 
Tabela 2. Estatísticas descritivas das medidas empregadas na amostra de Perdões $(n=215)$

\begin{tabular}{lcc}
\hline Medidas & Média & Desvio-Padrão \\
\hline Raven & 31,57 & 10,88 \\
Subteste informaçai (WISC III) & 14,34 & 4,12 \\
Questionário de informação geral e atual & 12,12 & 3,75 \\
Sinceridade & 27,55 & 4,03 \\
Neuroticismo & 30,78 & 3,74 \\
Psicoticismo & 18,98 & 2,53 \\
Extroversão & 41,33 & 3,17 \\
\hline
\end{tabular}

QIG: questionário de informação geral e atual; S: sinceridade; N: neuroticismo; P: psicoticismo; E: extroversão.

\section{Path models}

Vários path models foram testados utilizando-se o programa estatístico AMOS v.6. O modelo base inclui três variáveis de personalidade (psicoticismo-P, extroversão-E, neuroticismo-N), inteligência (Raven e Informação do WISC III) e idade como preditores da aquisição de informação geral e atual (Q|G). Nessa base de dados, presume-se que (a) a idade prediz inteligência e personalidade; (b) a idade prediz informação geral; (c) dimensões de personalidade estejam associadas à inteligência, e (d) a inteligência e a personalidade de forma independente, e sem influência da idade, predizem a aquisição da informação geral e atual.

O modelo final (Figura 1), extraindo todos os pesos não significativos, indica que o efeito da idade sobre inteligência é de 0,30; sobre psicoticismo é de $-0,17$ e sobre QIG é de 0,50. A correlação entre E e P (eliminando o efeito da idade em $\mathrm{P}$ ) é de -0,19. A corre-

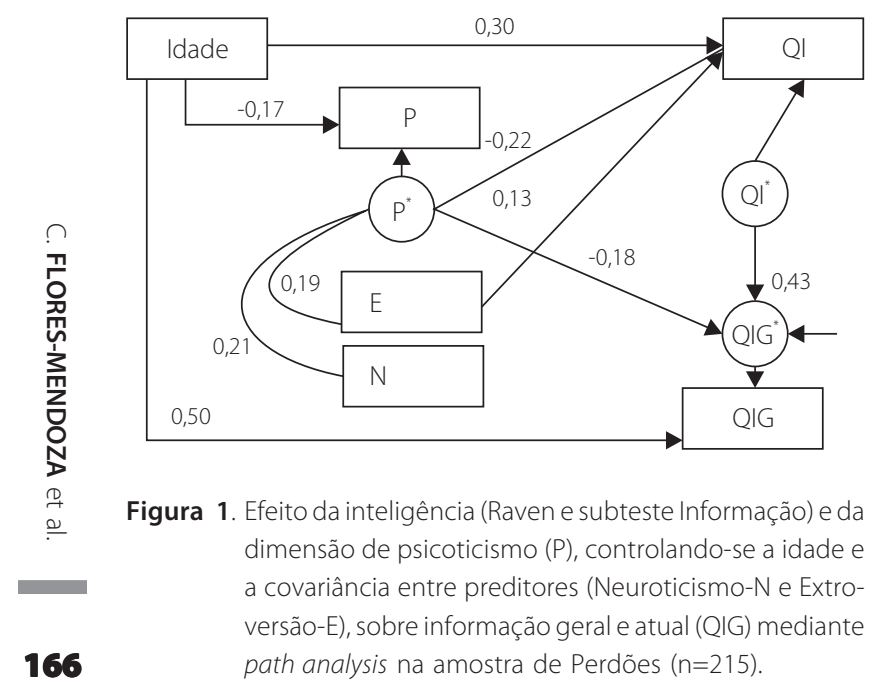

lação entre N e P (eliminando o efeito da idade em $P$ ) é de 0,21. O efeito de $P$ sobre inteligência é de -0,22. Finalmente, o efeito da inteligência sobre aquisição de informação geral e atual é de 0,43 (eliminando o efeito de $P$, E e da idade em inteligência assim como o efeito da idade em QIG); enquanto o efeito de P sobre o QIG é de -0,18. Os índices de ajuste do modelo foram adequados: $\mathrm{RMSEA}=0,067 ; \mathrm{TLI}=0,923$. Tais resultados indicam que, após a inteligência, a dimensão $P$ seja o único fator de personalidade que prediz a aquisição de informação geral e atual.

\section{Discussão}

Os resultados da investigação mostram vários pontos de interesse. Em primeiro lugar, existe uma associação positiva e moderada entre a inteligência, medida pelo teste Raven, e a aquisição informal de conhecimentos gerais e atuais, medida pelo QIG $(r=0,447)$. Em segundo lugar, essa associação persiste mesmo controlando-se o efeito da vulnerabilidade social da escola $(r=0,431)$ e é similar para meninos e meninas. Tais resultados significam que, apesar de haver maior nível intelectual e maior nível de conhecimentos gerais em crianças que frequentam escolas com menor risco de desamparo social, em termos gerais, há um efeito genuíno e independente da inteligência sobre o nível de aquisição espontânea de informação geral e atual.

Crianças com maior nível intelectual procuram informar-se do que ocorre no país e no mundo e, portanto, encontram-se mais atualizadas que seus pares de menor nível intelectual. No presente estudo, a inteligência dos escolares $\left(0,431^{2}=18,5 \%\right)$ explicou nove vezes mais a variação na aquisição informal de conhecimentos gerais do que a vulnerabilidade social da escola em que estavam matriculados $\left(0,142^{2}=2,01 \%\right)$.

Em terceiro lugar, os dados da segunda amostra mostraram que, após o controle do efeito desenvolvimental (efeito da idade), a inteligência e a personalidade (especificamente psicoticismo) apresentam de forma independente predição da informação geral e atual $(\mathrm{Q} \mid \mathrm{G})$, porém em intensidade variada. No caso, a inteligência $(0,43)$ prediz quase 2,5 vezes a mais do que a personalidade $(-0,18)$.

Esses resultados são semelhantes aos de estudos que compararam simultaneamente medidas de inteligência e personalidade ao desempenho escolar em 
crianças e adolescentes, como, por exemplo, os estudos de Di Fabio e Busoni (2007), cujos resultados mostraram que a inteligência sozinha pode explicar entre 4 e 17\% da variância no sucesso escolar, e, quando características de personalidade são adicionadas ao modelo, a explicação da variância pode aumentar entre 10 e 20\%. Mostra-se assim que características de personalidade acrescentam informação adicional à inteligência na predição do sucesso escolar.

No presente estudo, as associações obtidas entre dimensões de personalidade são aquelas encontradas na literatura. Assim, as dimensões E e N não são completamente independentes de $\mathrm{P}$, pois existe uma pequena associação entre elas que não está vinculada à idade; também E e P não são completamente independentes de inteligência. Parece existir uma leve associação entre essas dimensões e a inteligência.

De forma geral, os resultados mostraram que além da inteligência, o psicoticismo, visto como uma dimensão relacionada à antissociabilidade, apresenta associação, negativa no caso, com o comportamento de aquisição de informação geral. Isto é, quanto maior o traço de psicoticismo, menor é o interesse do indivíduo em saber o que ocorre no país e no mundo e, provavelmente, menor será o desempenho acadêmico e profissional.

O presente estudo tem a limitação de não ter medidas sobre hábitos de leitura em casa e/ou nível educacional dos pais, fatores que poderiam influenciar os valores de associação entre QIG com a inteligência e a personalidade. Deve-se recordar, porém, que o estudo de Colom e Flores-Mendoza (2007) mostrou não haver efeito do poder aquisitivo ou do nível de instrução dos pais no desempenho escolar em crianças de Belo Horizonte. Por último, o estudo não dispõe de medidas de memória, um construto provavelmente também associado à evocação de fatos nacionais ou mundiais. Na adaptação brasileira do WISC III, os subtestes Informação e Dígitos (memória a curto prazo) associaram-se em 0,46, e, na versão americana, a associação foi de 0,34 (Wechsler, 2002). É possível, portanto, que a memória seja um fator a ser considerado, apesar de o estudo de Hambrick et al. (in press) com pessoas adultas ter mostrado não haver efeito da memória sobre o conhecimento anterior ou atual. De qualquer forma, as três limitações que aqui se expõem devem ser controladas em futuras investigações.
Conclui-se que o conhecimento geral e atual parece refletir em primeiro lugar uma extensão da inteligência, isto é, uma "amplitude do intelecto" na classificação de Thorndike. A amplitude é o número de diferentes coisas que um indivíduo conhece e à qual toda pessoa em princípio teria acesso, como, por exemplo, informação geral (Jensen, 1998). Em segundo lugar, o conhecimento informal parece sofrer influência da personalidade, especificamente daquela dimensão que impele as pessoas a sensibilizarem-se e a acompanharem os fatos que ocorrem no seu ambiente social. Essa dimensão chamada de psicoticismo na teoria de Eysenck foi reclamada pelo próprio autor como uma dimensão geral que encobre Conscienciosidade e Amabilidade (Eysenck, 1992), justamente as dimensões que aparecem na literatura como preditores do desempenho acadêmico (Chamorro-Premuzic \& Furnham 2003).

Manter-se atualizado do mundo que nos rodeia é o preâmbulo da aquisição do "information literacy", um conceito relacionado à permanente aprendizagem e busca de informação relevante por parte das pessoas (Dudziak, 2003) e que é bastante necessário nas sociedades cujas economias estão baseadas em conhecimento (O'Sullivan, 2002). Nesse sentido, o presente estudo indicou que ambos os construtos psicológicos, inteligência e personalidade, parecem ser bastante importantes para explicar o porquê as pessoas desde cedo diferem no seu nível de informação geral e atual.

\section{Referências}

Angelini, A. L., Alves, I. C. B., Custódio, E. M., Duarte, W. F., \& Duarte, J. L. M. (1999). Padronização brasileira das matrizes progressivas coloridas de Raven. In J. C. Raven. Manual matrizes progressivas coloridas de Raven:escala especial. São Paulo: Centro Editor de Testes e Pesquisas em Psicologia.

Beier, M. E., \& Ackerman, P. L. (2001). Current events knowledge in adults: an investigation of age, intelligence and non-ability determinants. Psychology and Aging, 16 (4), 615-628.

Beier, M. E., \& Ackerman, P. L. (2003). Determinants of health knowledge: an investigation of age, gender, abilities, personality, and interests. Journal of Personality and Social Psychology, 84 (2), 439-448.

Beier, M. E., \& Ackerman, P. L. (2005). Age, ability, and the role of prior knowledge on the acquisition of new domain knowledge: promising results in a real-world learning environment. Psychology and Aging, 20 (2), 341-355. 
Brody, N. (1992). Intelligence. San Diego: Academic Press.

Browne, M. W., Cudeck, R., Tateneni, K., \& Mels, G. (2004). CEFA: Comprehensive exploratory factor analysis, (version 2). [Computer software and manual]. Available from http:// quantrm2.psy.ohio-state.edu/browne/

Bryman, A., \& Cramer, D. (1990). Quantitative data analysis for social scientists. London: Routledge.

Chamorro-Premuzic, F., \& Ackerman, P. (2006). Ability and personality correlates of general knowledge. Personality and Individual Differences, 41 (3), 419-429.

Chamorro-Premuzic, T., \& Furnham, A. (2003). Personality predicts academic performance: evidence from two longitudinal university samples. Journal of Research in Personality, 37 (4), 319-338.

Colom, R., \& Flores-Mendoza, C. E. (2007). Intelligence predicts scholastic achievement irrespective of SES factors: evidence from Brazil. Intelligence, 35 (3), 243-251.

De Raad, B., \& Shouwenburg, H. C. (1996). Personality in learning and education. European Journal of Personality, 10 (5), 303-336.

Deary, I. J., Whitman, M. C., Starr, J. M., Whalley, L. J., \& Fox, H. C. (2004). The impact of childhood intelligence on later life: following up the Scottish Mental Surveys of 1932 and 1947. Journal of Personality and Social Psychology, 86 (1), 130-147.

Di Fabio, A., \& Busoni, L. (2007). Fluid intelligence, personality traits and scholastic success. Empirical evidence in a sample of Italian high school students. Personality and Individual Differences, 43 (8), 2095-2104.

Dudziak, E.A. (2003). Information literacy: princípios, filosofia e prática. Ciência da Informação, 31 (1), 23-35.

Eysenck, H. J. (1992). A reply to Costa and McCrae. P or A and $\mathrm{C}$ - the role of theory. Personality and Individual Differences, 13 (8), 867-868.

Eysenck, H. J., \& Eysenck, S. B. G. (1975). Manual of the eysenck personality questionnaire. London: Hodder and Stoughton.

Gottfredson, L. (2003). Jobs and life. In H. Nyborg (Ed.), The scientific study of general intelligence (pp.293-342). Amsterdam: Pergamon.

Guerin, D. W., Gottfried, A. W., Oliver, P. H., \& Thomas, C. W. (1994). Temperament and school functioning during early adolescence. Journal of Early Adolescence, 14 (2), 200-225.

Hambrick, D. Z., Pink, J. E., Meinz, E. J., Pettibone, J. C., \& Oswald, F. L. (2008). The roles of ability, personality, and interests in acquiring current events knowledge: a longitudinal study. Intelligence, 36 (3), 261-278.

Heaven, P. C. L., Mak, A., Barry, J., \& Ciarrochi, J. (2002). Personality and family influences on adolescent attitudes to school and self-rated academic performance. Personality and Individual Differences, 32 (3), 453-46

Heaven, P. C. L., Ciarrochi, J. R., \& Vialle, W. (2007). Conscientiousness and Eysenckian Psychoticism as predictors of school grades. A one-year longitudinal study. Personality and Individual Differences, 42 (3), 535-546.

Jensen, A. R. (1998). The $g$ factor. London: Praeger.

Laidra, K., Pullman, H., \& Allik, J. (2007). Personality and intelligence as predictors of academic achievement: A cross-sectional study from elementary to secondary school. Personality and Individual Differences, 42 (3), 441-451.

Lubinski, D. (2004). Introduction to the special section on cognitive abilities: 100 years after spearman's (1904) 'General intelligence', objectively determined and measured". Journal of Personality and Social Psychology, 86 (1), 96-111.

Mansur-Alves, M. (2007). Diferenças individuais na dimensão neuroticismo em população escolar por meio dos testes BFQ-C e EPQ-J. Dissertação de mestrado não-publicada, Universidade Federal de Minas Gerias, Belo Horizonte.

Martin, R. P., Drew, K. D., Gaddis, L. R., \& Moseley, M. (1988). Prediction of elementary school achievement from preschool temperament: three studies. School Psychology Review, 17, (1), 125-137.

Nahas, M. I. P., Ribeiro, C. A., Esteves, O. A., Moscovitch, S., \& Martins, V. L. A. B. (2000). O mapa da exclusão social de Belo Horizonte: metodologia de construção de um instrumento de gestão urbana. Caderno de Ciências Sociais, 7 (10), 75-88.

O'Sullivan, C. (2002). Is information literacy relevant in the real world? Reference Services Review, 30 (1), 7-14.

Organization for Economic Co-operation and Development. (2001). Knowledge and skills for life. First results from PISA 2000. Paris: OECD.

Oleto, R. R. (2006). Percepção da qualidade da informação. Ciência da Informação, 35 (1), 57-62.

Petrides, K. V., Chamorro-Premuzic, T., Frederikson, N., \& Furnham, A. (2005). Explaining individual differences in scholastic behaviour and achievement. British Journal of Educational Psychology, 75 (2), 239-255.

Raven, J. (2001). Matrizes progressivas de Raven: escala geral. Rio de Janeiro: Centro de Psicologia Aplicada.

Roid, G. H. (2003). Stanford-binet intelligencescales, fifth edition, technical manual. Rolling Meadows, IL: Riverside Publishing.

Slobodskaya, H. R., Safronova, M. V., \& Windle, M. (2005). Personality, temperament and adolescent adjustment in modern Russia. Personality and Individual Differences, 39 (1), 167-78

Wechsler, D. (2002). Escala de inteligência Wechsler para crianças (3a ed.). São Paulo: Casa do Psicólogo.

Recebido em: 3/4/2008

Versão final reapresentada em: 18/6/2009

Aprovado em: 29/1/2010 